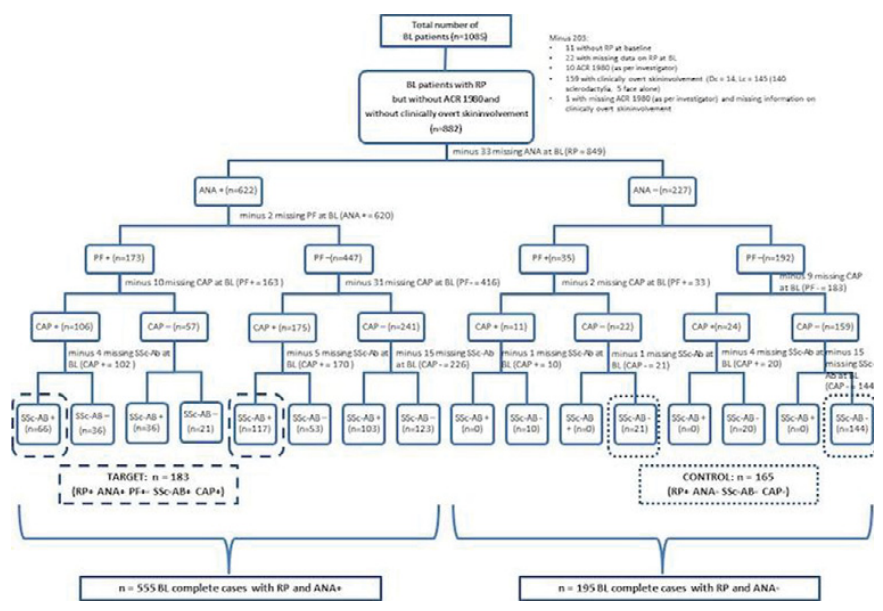

Conclusions: The VEDOSS cohort presents predominantly an "Early" NVC SSC pattern. Notably the prevalence of NVC SSc patterns was higher in the ANA+ than ANA- stratum. This evidence further reflects the pivotal role of NVC in the very early diagnosis of SSC.

Disclosure of Interest: None declared

DOI: 10.1136/annrheumdis-2017-eular.2932

\section{OP0036 SEROLOGICAL BIOMARKERS OF ECM TURNOVER ARE ASSOCIATED WITH SKIN FIBROSIS AND LUNG INVOLVEMENT IN SYSTEMIC SCLEROSIS}

S. Kubo ${ }^{1}$, A.S. Siebuhr ${ }^{2}$, A.-C. Bay-Jensen ${ }^{2}$, P. Juhl ${ }^{2}$, M.A. Karsdal ${ }^{2}$, K. Nakano ${ }^{1}$, S. Nakayamada ${ }^{1}$, Y. Tanaka ${ }^{1} .{ }^{1}$ The First Department of Internal Medicine, University of Occupational and Environmental Health, Japan, Fukuoka, Japan; ${ }^{2}$ Nordic Bioscience, Biomarkers and Research, Herlev, Denmark

Background: Extracellular matrix (ECM) unbalance of the skin is a hallmark of systemic sclerosis (SSc). However, currently there is no objective tool to monitor the ECM unbalance in SSc patients allowing for better understanding of the disease stage and activity.

Objectives: We investigated the potential of serological biomarkers of ECM turnover (collagen formation and degradation) as biomarkers of skin fibrosis and internal organ involvement in SSc patients.

Methods: Peripheral blood obtained from 79 SSc patients and 19 healthy subjects was included in the study. Type I, III, IV, V and VI collagen formation (P1NP, PRO-C3, P4NP7S, PRO-C5, PRO-C6) and degradation (C3M, C4M2, C5M, C6M) biomarkers were detected by ELISA in serum. Modified Rodnan skin score (mRSS) and extent of internal organ involvement (renal, lung, vasculopathy and gastrointestinal) were recorded for SSc patients with a scoring between 0 and 4 , with 0 being no involvement and 4 being severe involvement. Mann-Whitney t-test was used to test difference in the biomarker levels between the patient groups and in groups with and without internal organ involvement. Spearman's correlation coefficient investigated the association between biomarkers and clinical manifestations.

Results: SSc patients had a mean age of 63.0 years, mean disease duration of 98.3 months and a mean mRSS of 11.1. SSc patients compared to healthy individuals had higher levels of C5M, C6M and PRO-C6 ( $p=0.0001, p<0.0001$, $p<0.0001$, respectively). The levels of type VI collagen formation and degradation (PRO-C6 and C6M) were twice as that of healthy controls (12.6 vs. 5.4 and 26.0 vs $16.7 \mathrm{ng} / \mathrm{ml}$, respectively). C4M2, PRO-C3 and PRO-C6 was associated with skin fibrosis assessed by mRSS (Spearman's rho $=0.24,0.39$ and 0.29 , respectively). Patients with signs and manifestation of lower gastrointestinal lesion compared to patients without lesion had higher levels of C3M (median $10.7 \mathrm{vs} 13.8 \mathrm{ng} / \mathrm{mL}$, $p=0.017$; figure). PRO-C6 was higher in patients with pulmonary hypertension compared to patients without any signs of pulmonary hypertension (median 9.6 vs $14.3 \mathrm{ng} / \mathrm{mL}, \mathrm{p}=0.006$ ) with a Spearman's correlation coefficient of 0.31 . C6M was higher in patients with definite pulmonary hypertension $(>1)$ compared to patients with signs of or no pulmonary hypertension (median $22.1 \mathrm{vs} 29.9 \mathrm{ng} / \mathrm{mL}$, $\mathrm{p}=0.026$ ). P1NP was lower in patients with interstitial pneumonia, whereas C6M

\begin{tabular}{lcccc}
\hline & Healthy & SSc & P-value & $\begin{array}{c}\text { Spearman's correlation to mRSS } \\
\text { Rho (p-value) }\end{array}$ \\
\hline C3M & 12.8 & 13.4 & - & $0.21(0.06)$ \\
C4M2 & 31.1 & 34.0 & - & $0.24(0.03)$ \\
C5M & 2.6 & 3.9 & 0.0001 & $0.03(0.81)$ \\
C6M & 16.7 & 26.0 & $<0.0001$ & $0.18(0.11)$ \\
P1NP & 136.9 & 130.4 & - & $0.03(0.80)$ \\
PRO-C3 & 14.2 & 20.8 & - & $0.39(<0.001)$ \\
P4NP7S & 286.8 & 290.3 & - & $0.19(0.10)$ \\
PRO-C5 & 525.2 & 484.4 & - & $0.07(0.52)$ \\
PRO-C6 & 5.4 & 12.6 & $<0.0001$ & $0.29(0.01)$ \\
\hline
\end{tabular}

was higher. There was no difference in the biomarker levels with and without upper gastrointestinal lesions, renal dysfunction or vasculopathy.
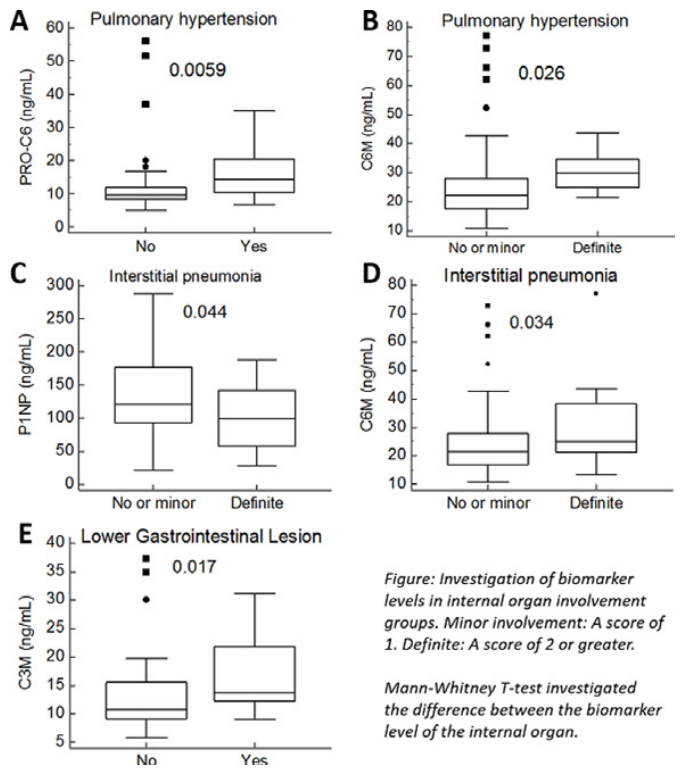

Conclusions: Serological biomarkers of type $\mathrm{V}$ and $\mathrm{VI}$ turnover (C5M, C6M, PRO-C6) were associated with SSc, but not serological biomarkers of type I, III and IV collagen. Especially, turnover of type VI collagen was associated with skin sclerosis, pulmonary hypertension, interstitial pneumonia and renal dysfunction. This study indicates that biomarkers of collagen turnover have a potential as new objective tool of skin fibrosis and internal organ involvement in SSc.

Disclosure of Interest: S. Kubo Speakers bureau: Bristol-Myers, A. S. Siebuhr Employee of: Nordic Bioscience, A.-C. Bay-Jensen Employee of: Nordic Bioscience, P. Juhl Employee of: Nordic Bioscience, M. A. Karsdal Employee of: Nordic Bioscience, K. Nakano: None declared, S. Nakayamada: None declared, Y. Tanaka Grant/research support from: Mitsubishi-Tanabe, Takeda, Daiichi-Sankyo, Chugai, Bristol-Myers, MSD, Astellas, Abbvie, Eisai, Speakers bureau: Abbvie, Chugai, Daiichi-Sankyo, Bristol-Myers, MitsubishiTanabe, Astellas, Takeda, Pfizer, Teijin, Asahi-kasei, YL Biologics, Sanofi, Janssen, Eli Lilly, GlaxoSmithKline DOI: 10.1136/annrheumdis-2017-eular.4855

\section{OP0037 EVALUATION OF SKIN INVOLVEMENT IN SYSTEMIC SCLEROSIS PATIENTS BY USING TWO ULTRASOUND TRANSDUCERS WITH DIFFERENT FREQUENCY}

B. Ruaro, A. Sulli, S. Paolino, C. Pizzorni, M. Ghio, E. Alessandri, A.C. Trombetta, C. Cosso, M. Cutolo. Research Laboratory and Academic Division of Clinical Rheumatology, Department of Internal Medicine, University of Genova, IRCCS AOU San Martino, Genoa, Italy

Background: The modified Rodnan skin score (mRSS) is the validated method to evaluate the extension of skin involvement in systemic sclerosis (SSc) and to distinguish between patients with limited cutaneous skin involvement (IcSSc, skin involvement is confined to the extremities) or diffuse (dcSSc) $(1,2)$. Recently several studies have demonstrated that skin high frequency ultrasound (US) is a valid and reproducible technique to measure dermal thickness (DT) in patients with SSc (3-6).

Objectives: To compare the values of DT obtained by two ultrasound transducers with different frequency (18 MHz and $22 \mathrm{MHz}$ ) in evaluating the DT in IcSSc patients and healthy controls.

Methods: Thirty-seven IcSSc patients (mean age 62 $\pm 13 S D$ years, mean disease duration $5 \pm 5$ SD years) and 37 healthy controls (CNT) sex and age matched were enrolled after informed consent. Both US transducers of 18 and $22 \mathrm{MHz}$ (Esaote, Genova) were used to evaluate DT in the seventeen areas of the skin (zygoma, fingers, dorsum of hands, forearms, arms, chest, abdomen, thighs, legs, feet) of SSc patients where Rodnan skin score (mRSS) is usually assessed. Skin US was also performed in the same seventeen areas of CNT, looking for DT differences in comparison with IcSSc patients. Statistical analysis was carried out by non parametric tests.

Results: DT evaluated with the $22 \mathrm{MHz}$ probe was found significantly higher in all body areas in comparison with the $18 \mathrm{MHz}$ transducer, both in IcSSc patients $(p<0.01)$ and in CNT $(p=0.05)$. The median difference of DT values between the two probes was of 0.11 millimetres in IcSSc patients (minimum 0.0023 , maximum $0.28 \mathrm{~mm}$ ) and 0.01 millimetres in CNT (minimum 0.0029, maximum $0.03 \mathrm{~mm}$ ). Of interest, in IcSSc DT evaluated by $18 \mathrm{MHz}$ transducer was recognized significantly higher $(p<0.001)$ also in four out of six skin areas where the mRSS was found normal (score $=0$ ) (upper-arms, chest and abdomen), with exclusion of thighs $(p=0.08)$, in contrast with the classification of IcSSc. However, by using the 22 